\title{
Inhaled Insulin - Update Addendum to previously published The Role of Emerging Insulin and Regimens in Overcoming Insulin Treatment Resistance on 1/29/2018
}

\author{
Steven H Barag1,2,3,4*, Talin Meshefedjian ${ }^{5}$, Kevin Diep ${ }^{5}$ and Andrew Wilson DO \\ ${ }^{1}$ Aureus Medical Group, Inc., Nebraska, USA \\ ${ }^{2}$ Western University College of Osteopathic Medicine, California, USA \\ ${ }^{3}$ Touro University College of Osteopathic Medicine, California, USA \\ ${ }^{4}$ San Antonio Regional Hospital, California, USA \\ ${ }^{5}$ OMS IV, Western University College of Osteopathic Medicine, California, USA
}

The newest and possibly most innovative insulin modality comes in an inhaled form. Initially approved by the FDA in 2006, inhaled insulin appears to be a promising alternative for patients with fear of needles and negative views of insulin therapy. The first inhaled insulin on the market in 2006 had mild success for patients who could not tolerate needles but did not gain widespread acceptance due to high dose administration, inefficient absorption, inflexible dosing and a bulky device. As of 2014, a new rapid acting inhaled insulin has been approved by the FDA that uses technosphere technology for more efficient absorption in the lungs, greater dosing flexibility and a small convenient delivery system [1]. Technosphere technology involves formulating insulin with the carrier molecule fumaryl diketopiperazine (FDKP). When inhaled into the acidic environment of the lungs, it assembles into small technospheres containing insulin for effective delivery and rapid absorption into the bloodstream. It will be referred to as technosphere insulin or TI for short for the remainder of the article [2].

Clinical trials are still ongoing for TI, but current literature suggests that TI is marginally less effective than subcutaneous insulin. Two of the most widely available randomized control trials published in 2015 made the following conclusions. The first randomized controlled trial (RCT) compared TI with aspart insulin therapy over 24 weeks monitoring hemoglobin A1c levels (HbA1c) and showed a mean reduction of $0.21 \%$ for TI and $0.4 \%$ for aspart [3]. Another RCT compared TI with placebo therapy for a duration of 24 weeks also monitoring HbAlc levels showed TI to be superior with a mean HbAlc reduction of $0.8 \%$ versus $0.4 \%$ for placebo [4]. A meta-analysis including 8 clinical trials looked at mean $\mathrm{HbA} 1 \mathrm{c}$ reduction comparing TI to subcutaneous insulin therapy showed similar results as above. Subcutaneous insulin was superior to $\mathrm{TI}$, showing a $0.16 \%$ greater decrease in HbA1c [5].

It is well known that the lungs are an effective site for medication delivery due to their large surface area and extensive alveolar-capillary network that facilitates drug absorption. Inhaled insulin takes advantage of this principle, however certain precautions must be considered for patient safety. The most common adverse effects of TI are cough and throat irritation that usually decrease over time and completely subside shortly after discontinuation of therapy according to clinical trials $[3,4]$. TI is contraindicated for use in patients with chronic lung disease including chronic obstructive pulmonary disease (COPD) and asthma due to increased risk of bronchospasm. It has been shown that TI causes a modest decline in lung function measured by the forced expiratory volume in one second $\left(\mathrm{FEV}_{1}\right)$ that occurs over the first 3 months and continues for the duration of treatment. The manufacturer of TI recommends pulmonary function tests be conducted before starting treatment, 6 months into therapy and annually thereafter, even in the absence of pulmonary symptoms. In addition, there has been higher incidence of diabetic ketoacidosis (DKA) and lung cancer while on TI than observed in comparators. The data is not sufficient to suggest TI is the cause, but benefits should always outweigh risks when considering TI therapy. The long-term safety of TI continues to be investigated [2].

The are several distinct advantages that TI has over conventional injectable insulin. It is easy and non-painful to administer, offers flexible dosing before meal or within 20 minutes after meal and decreases postprandial hypoglycemia and weight gain compared to subcutaneous insulin [6]. This is mainly owed to the drug's relatively short half-life of approximately 30 minutes. It causes less postprandial hypoglycemia and as a result, patients are not as likely to overeat and gain weight after its administration [2,5]. TI is indicated for patients with type 1 and 2 diabetes on basal insulin, type 2 diabetes poorly controlled with oral agents, needle phobia or preference for meal time insulin in an inhaled form. Other indications also include patients who develop late post-prandial hypoglycemia (4-5 hours after meal) and skin reactions to subcutaneous insulin injections [6]. Overall, TI presents a novel and useful approach to insulin therapy for the select patient, especially in the context of PIR.

\section{References}

1. Mohanty R, Das S (2017) Inhaled Insulin - Current Direction of Insulin Research. $J$ Clin Diagn Res 11: OE01-OE02. [Crossref]

2. Affreza. Prescribing Information. MannKind Corporation. Danbury, CT, June 2014

3. Bode BW, McGill JB, Lorbe DL, Gross JL, Chang PC, et al. (2015) Inhaled Technosphere Insulin Compared with Injected Prandial Insulin in Type 1 Diabetes: A Randomized 24- Week Trial. Diabetes Care 38: 2266-2273. [Crossref]

4. Rosenstock J, Franco D, Korpachev V, Shumel B, Ma Y, et al. (2015) Inhaled Technosphere Insulin Versus Inhaled Technosphere Placebo in Insulin-Naïve Subjects with Type 2 Diabetes Inadequately Controlled on Oral Antidiabetes Agents. Diabetes Care 38: 2274-2281. [Crossref]

${ }^{\star}$ Correspondence to: Steven H. Barag, 4794 Haven Ave. \#250 Rancho Cucamonga, CA, 91730 USA, Tel: (909) 240-5285; E-mail: drbarag@gmail.com

Received: May 06, 2018; Accepted: May 18, 2018; Published: May 21, 2018 
Barag H (2018) Inhaled Insulin - Update Addendum to previously published The Role of Emerging Insulin and Regimens in Overcoming Insulin Treatment Resistance on $1 / 29 / 2018$

5. Pittas AG, Westcott GP, Balk EM (2015) Efficacy, safety, and patient acceptability of Technosphere inhaled insulin for people with diabetes; a systematic review and metaanalysis. Lancet Diabetes Endocrinol 3: 886-894. [Crossref]
6. Mikhail N (2016) Place of technosphere insulin in treatment of diabetes. World $J$ Diabetes 7: 20:599-604. [Crossref]

Copyright: (C2018 Barag H. This is an open-access article distributed under the terms of the Creative Commons Attribution License, which permits unrestricted use, distribution, and reproduction in any medium, provided the original author and source are credited. 